\title{
TTR
}

Traduction, terminologie, re?daction

\section{Michel Ballard. De Cicéron à Benjamin. Lille, Presses \\ Universitaires de Lille, 1992.}

\section{André Lefevere}

Volume 6, numéro 2, 2e semestre 1993

Traduction, mixité, politique

URI : https://id.erudit.org/iderudit/037156ar

DOI : https://doi.org/10.7202/037156ar

Aller au sommaire du numéro

\section{Éditeur(s)}

Association canadienne de traductologie

ISSN

0835-8443 (imprimé)

1708-2188 (numérique)

Découvrir la revue

Citer ce compte rendu

Lefevere, A. (1993). Compte rendu de [Michel Ballard. De Cicéron à Benjamin.

Lille, Presses Universitaires de Lille, 1992.] TTR, 6(2), 163-168.

https://doi.org/10.7202/037156ar d'utilisation que vous pouvez consulter en ligne.

https://apropos.erudit.org/fr/usagers/politique-dutilisation/ 


\section{Michel Ballard. De Cicéron à Benjamin. Lille, Presses Universitaires de Lille, 1992.}

Le fait que l'on publie à nouveau des aperçus historiques de la traduction est probablement le témoignage le plus éloquent de l'intérêt grandissant pour la traduction dans le monde universitaire. Le présent ouvrage, sous-titré «Traducteurs, Traductions, Réflexions» et publié trois ans après l'Imitatio du regretté Frederick Rener, est paru presque en même temps que les deux premiers volumes qu' Hans Vermeer a consacrés à l'histoire de la traduction sous le titre Skizzen zu einer Geschichte der Übersetzungswissenschaft. Par surcroît, la FIT s'apprête à publier, par les bons offices de Jean Delisle et de Judith Woodsworth, une histoire thématique de la traduction en 1994. Ces ouvrages vont prendre la relève d'histoires plus anciennes, comme celles de Kelly et de Van Hoof, et de chapitres consacrés à l'histoire de la traduction dans les ouvrages publiés par Steiner, Bassnett, Nida, Mounin et Savory que Michel Ballard énumère dans son introduction.

Le souci d'histoire est sans doute inspiré par la volonté d'établir une fois pour toutes (comme si cela était possible, mais il faut persévérer) l'importance de la traduction comme phénomène culturel. Citons l'auteur: "Face au mépris avec lequel on traite souvent une activité considérée comme secondaire dans le domaine littéraire, il s'agissait d'abord d'en affirmer la présence massive et constante» (p. 11). Mais les histoires de la traduction ont une tout autre importance parmi ceux qui s'occupent déjà de la traduction, qui la font ou qui l'enseignent: elles font ressortir de façon définitive la contingence des «normes» de la traduction. L'histoire inspire l'humilité, particulièrement en rappellant que les normes ont changé et continuent de changer. 
Les chapitres dans le livre de Michel Ballard se succèdent par ordre chronologique et le premier chapitre remonte à ce que l'on pourrait appeler «l'aube" même de la traduction en Occident: les efforts pédagogiques et lexicographiques des Assyriens et des Babyloniens, ainsi que les efforts philologiques de la civilisation suméro-akkadienne. Le monde des savants qui s'occupent de la traduction saura gré à Michel Ballard d'être remonté aussi loin dans le temps, puisque «l'opinion reçue» en Occident laisse souvent commencer la tradition de la traduction avec la traduction de l'Odyssée en Latin par Livius Andronicus au deuxième siècle avant l'ère chrétienne.

Il nous est manifestement impossible de "résumer" le livre de Michel Ballard ici, et nous nous proposons donc d'en extraire les éléments qui nous ont frappé, non seulement parce que nous ne les connaissions pas, ou pas dans un tel détail, mais parce que leur importance ultérieure s'est avérée considérable.

Commençons par Philon (dit «le Juif») d'Alexandrie qui considère la traduction comme révélation, et qui a exercé une influence remarquable sur le Moyen Âge, influence qui s'étend d'ailleurs à Luther qui, lui aussi, considérait le traducteur (qui s'appellait Martin Luther, par un heureux, ou même divin hasard) comme un instrument de Dieu. Michel Ballard démontre ensuite que c'est à Philon qu'on doit aussi la notion du littéralisme en traduction de textes sacrés, littéralisme qui s'est ensuite - connexion que Michel Ballard ne fait pas - appliqué aussi aux textes profanes considérés comme produits par des êtres dits «géniaux» depuis l'ère romantique.

Entre Philon et le problème de la dominance culturelle des langues considérées comme «sacrées» la distance n'est pas grande. En Occident les langues considérées comme "sacrées» étaient surtout l'hébreu, ensuite le grec, et même le latin. Les conséquences de l'existence de langues perçues comme sacrées furent importantes: "l'idée que les langues nouvelles, les vernaculaires, les langues rattachées à une civilisation inférieure, n'ont pas le même pouvoir que d'autres» (p. 36). Cette croyance a eu pour autres conséquences non seulement l'idée de l'impossibilité de la traduction, mais encore celle de la traduction comme activité dégradante, puisqu'elle partait 
des langues sacrées pour aboutir aux langues considérées «inféricures».

Le traitement de Jérôme dans l'ouvrage de Ballard est un mélange admirable de l'usage de sources antérieures et du jugement de l'auteur lui-même. Ballard attire l'attention du lecteur sur le caractère fondamentalement «justificatif» de la "lettre à Pammachius», en remarquant que la justification sera «la motivation principale de bon nombre de préfaces ultérieures» (p. 46), laissant apercevoir les rapports (très inégaux, évidemment) entre la traduction et le pouvoir, sans toutefois les étudier de façon plus approfondie dans le reste de son livre. Mais on ne peut pas lui en tenir rigueur, puisqu'il a fait tant d'autres choses avec une verve et une érudition remarquables, et puisqu'il reste tant d'autres choses à faire. Il aurait peut-être pu faire ressortir de façon plus claire que cette attitude justificative a, pendant de longs siècles, découragé une étude plus objective du phénomène de la traduction en Occident. La note (47) que consacre Ballard à la filiation malheureuse JérômeStörig-Berman (une traduction erronce qui se trouve dans le livre Das Problem des Übersetzens qu'a édité Störig et qui est devenue la base $d^{\prime} u n e$ argumentation forcément erronée, elle aussi, dans le passage que le regretté Antoine Berman a consacré à Luther dans l'Épreuve de l'étranger) constitue un véritable pas en avant en matière d'histoire de la traduction, tout comme l'évaluation lucide et pénétrante que consacre l'auteur à la contradiction fondamentale qui est à la base de l'ouvrage "justificatif» de Jérôme: «une contradiction qui lui fait défendre un style de traduction libre d'où il exclut les textes religieux alors que ses exemples sont tirés de textes religieux» (p. 55).

La partie la plus éclairante de l'ouvrage de Michel Ballard traite du Moyen Âge. Ballard commence par mettre en évidence la différence entre l'Angleterre médiévale, où l'on s'écarte plus volontiers du littéralisme dominant par souci de clarté (l'auteur cite les traductions faites par Alfred le Grand et par Aelfric) et le reste de l'Europe où l'on traduit de façon plus "prudente».

Les passages qui traitent de l'école de traduction de Bagdad sont d'une importance capitale en ce qu'elles dépassent un eurocentrisme considéré non seulement normal, mais même naturel dans la plupart des ouvrages antérieurs consacrés à l'histoire de la 
traduction. La description de l'école de Bagdad, et surtout du rôle prépondérant qu'ont joué les bibliothèques, tant publiques que privées, dans l'entreprise de la traduction axée sur cette école au neuvième siècle de notre ère commune, sert admirablement à mettre en évidence l'écart énorme entre le monde islamique médiéval et $l^{\prime}$ Europe, où la première bibliothèque publique fut fondée, comme nous le rappelle Ballard, par Cosme de Médicis en 1442, au moins quatre siècles plus tard.

L'ouvrage de Ballard est remarquable à bien des égards, dont le fait de mettre en évidence le rôle joué par les mécènes n'est certainement pas le moindre. Il consacre des pages éclairantes aux contacts entre le grec et le latin en Europe médiévale, et aussi aux contacts entre l'arabe et le latin. Le rôle joué par le roi Alphonse $X$ au cours de l'entreprise traductrice de Tolède est dégagé, tout comme les mérites des rois de France Charles V et Charles VIII, du pape Nicolas $V$, et des princes Médicis Cosme et Laurent pour la traduction du latin vers les langues dites alors vernaculaires d'Europe.

Ballard met en relief l'épître dédicatoire à la traduction de Valère le Grant par Simon de Hesdin, membre du groupe de traducteurs dont s'était entouré Charles $V$, sans doute parce qu'on peut y trouver ce qui pourrait bien être un des premiers énoncés consacrés au public auquel était destiné la traduction. Simon de Hesdin " $s$ 'excuse de ne pas faire les digressions habituelles car traduisant pour des gens lais qui sont pressés et veulent aller directement aux faits, il s'est senti obligé d'adopter un style plus précis» (p. 86). Ballard relève le contraste entre cette attitude alors isolée et l'attitude répandue au Moyen Âge caractérisée toujours par la dominance du complexe d'infériorité à l'endroit de la traduction et des "vernaculaires", infériorité qui doit précisément être "compensée" par la rhétorique du traducteur comme une sorte de "valeur ajoutée».

En passant du Moyen Âge à la Renaissance, Ballard "démystifie" les deux coryphées traditionnels de la proto-traductologie française du temps de la Renaissance, en consacrant quelques pages fascinantes au procès d'Estienne Dolet, et en révélant que la Défence et Illustration de Du Bellay est un démarquage du Dialogue des langues publié par Sperone Speroni à 
Venise en 1542. Cela ne diminue évidemment en rien l'influence énorme de l'ouvrage de Du Bellay sur la pratique de la traduction en France jusqu'à l'ère des Belles Infidèles. Selon Ballard, «en posant le principe platonicien de l'inspiration divine, Du Bellay fait basculer la traduction de la poésie dans l'impossible ou presque» (p. 122). De façon plus générale, il "relègue la traduction à un rang second" (p. 123).

Ballard fait justice au problème de la traduction de la Bible à l'époque de la Renaissance en lui consacrant bon nombre de pages. Il met en évidence la traduction du Nouveau Testament par Erasme, qui uprend soin de dire que la Vulgate doit continuer d'être lue dans les églises et les écoles et que sa traduction est pour les savants et les étudiants de théologie» (p. 139). La distinction faite par Érasme met en valeur la distinction ultérieure entre les traductions littéraires (et non pas religieuses) faites pour les lettrés, et donc sans grand souci d'informer, qui dominent en Europe jusqu'au milieu du dix-huitième siècle, et les traductions faites depuis pour un public ignorant les langues originelles, et donc de nature surtout «informative».

Si Ballard n'a pas fait ce rapprochement, par contre en parlant de la traduction de la Bible par Luther il rapproche le réformateur allemand du vingtième siècle, et plus particulièrement des théories de Nida, en décrivant sa façon de traduire comme «traduction dynamique».

Enfin, la discussion de Malherbe et de son influence sur la formation de la doctrine des Belles Infidèles est fort éclairante, tant en soulignant l'identification du «beau» avec le «français» opérée par Malherbe dans ses écrits, et la diminution de l'écart entre la prose et la poésie, qui devient, par voie de conséquence, plus «traduisible».

Les deux derniers chapitres de l'ouvrage nous ont semblé moins révélateurs, mais nous ne doutons pas qu'ils le soient pour le lecteur et la lectrice découvrant l'histoire de la traduction pour la première fois. Pour conclure, on pourrait aussi poser l'éternelle question de l'universalité des tentatives d'histoire de la traduction. Celle de Kelly a été accusée d'être «trop anglaise»; celle de Ballard serait-elle «trop française»? Il nous semble que Ballard a fait ce qu'il a pu, dans l'état actuel de nos connaissances historiques. Il a d'ailleurs fait un usage extrêmement intelligent et productif des 
sources énumérées dans les notes et dans la bibliographie. Le fait demeure que le chercheur sait toujours un peu plus de la tradition propre à sa culture d'origine, et nous ne voyons pas en quoi cela serait répréhensible, surtout si le chercheur en question utilise ses connaissances d'une manière aussi éclairante que l'a fait Michel Ballard.

André Lefevere

University of Texas at Austin 